\title{
DRUG RESISTANCE OF BACTERIA: A GLOBAL CHALLENGE
}

\section{Manish Wasamwar' ${ }^{1}$, Vijay Wadhai ${ }^{1}$, Gopal Gond ${ }^{2}$}

\author{
1- Sardar Patel Mahavidyalaya, Chandrapur
}

2- Guru Nanak Science College, Ballarpur, Dist- Chandrapur

Email: manishwasamwar@gmail.com

\begin{abstract}
Multidrug resistant urinary tract infection is a most problematic infection and it is very difficult to doctors to manage the patient by using appropriate antibiotics due to development of multidrug resistant strains of bacteria. MDR strains shows resistant to maximum number of antibiotics and sensitive to very few antibiotics. ESBL producing eneterobacteriaceae have traditionally been treated with carbapenems. Now a day the infection with MDR $P$. aeruginosa is treated with colistin and MDR Acinetobacter is treated colistin and tigecycline. The review is focus on the study of mechanism of resistant particular antibiotics and emerging multidrug resistant amongs bacteria causing urinary tract infection.
\end{abstract}

Keywords : Urinary tract infection, multidrug resistant, antibiotic, bacteria

\section{INTRODUCTION:}

The bacterial associated Urinary Tract infection is most common infection found in all ages and both the genders offering antibiotic resistance in most of the cases of infection. (Stamm WE, 2001). Now a day's antibiotic resistance has becomes major public health problem leads to increase resistant to huge number of antibiotics from few years. The antibiotics are also responsible to develop multidrug resistant strain due to resistance genes of resistant bacteria .The rapid emergence of antibiotic resistant transfer genes among uro-pathogens are important cause of development of antibiotic resistance. (Stuart B., 2002). The increased antibiotic resistant suggest that the choice of antibiotic should be guided by culture and sensitivity assay. In past decades many kind of resistant strains are discovered. Example Methicillin Resistant Staphylococcus aureus (MRSA) (Wagenlehner FME, 2004), Multidrug resistant Pseudomonas aeroginosa (Linuma Y, 2007). 
Vancomycin resistant Entercocci (VRE) (Gold HS; 2001), Extended spectrum beta-lactamase (ESBL) resistant enterococci. (Bhattacharya S: 2006)

\section{PRINCIPLE OF ANTIMICROBIAL THERAPY}

The objective of antibacterial therapy is to eliminate the bacterial growth in the urinary tract and the antibiotics used are efficacious, safe, and cost effective. The basic c treatment starts with the antibiotics which are number of choices available in market. The resolution of infection is dependent on the susceptibility of the bacteria to the concentration of the antibiotic achieved in urine. Antibiotics should eliminates the bacterial growth in urinary tract when proper antibiotics are used which is able to achieve level in urine and the duration that this level remains above the minimum inhibitory concentration (MIC) of microorganism that attack on urinary tract. (Hooton TM, 1991). An effective antibacterial usually achieved MIC both in serum and urine of healthy adults, the urinary level often many folds greater than serum level. However the serum levels are critical in patients with uro-sepsis and urinary tract infection involving renal parenchyma. (Jacobson SH, 1991)

\section{MECHANISM OF RESISTANCE OF ANTIBIOTICS IN MULTIDRUG RESISTANT URINARY PATHOGENS}

Urinary tract isolates have developed several mechanisms of resistance to currently used antibiotics, including B-lactamases, efflux pumps, porin mutation, modifying enzymes and binding site mutations. In addition, horizontal transfer of combined resistance to multiple drugs is partially responsible for the rapid immergence of resistance in human. The rapid immergence of resistance is due to the resistance genes associated in clusters and transferred together. Horizontal transfer is a mechanism of transmission and dissemination of multiple drug resistance among bacterial pathogens. (Infect. Dis. Societ. of America). The awareness of the most relevant pattern of 
resistance among bacteria is not only on the basis of administration of antibiotics, but also for the development of new antimicrobial compounds aiming to overcome specific resistant determinants.

\section{Resistance to B-Lactams}

Urinary bacteria may develop several mechanisms of resistance to Blactams which is most efficient method of resistance to these agents in Gramnegatives is the synthesis of B-lactamases. B- lactamases have presumably evolved to fight natural B-lactams, produced by bacteria such as Streptomyces, Lysobacter, penicillium or Acremonium. (Brakhage AA, 2005). However the widespread administration of antibiotics is influenced the development of B- lactamases mediated resistance. Since B- lactam antibiotics came into clinical use, B-lactamases are evolved with them. One of the first B-lactamases able to confirm resistance to B-lactam known as TEM I develop in the sixties after the widespread use of ampicilin in human medicine. (Rawat D, 2010). The emergence of MBL mediated resistant among enterobacteriacae has also becomes serious health concern. More ever the increase in international travel is likely to be contributory factor for the ascendancy of mobile MBL genes as much as the mobility among individual bacteria. A new plasmidic MBL, New Delhi MBL called NDM I. Has been recently identified in $K$. pneumonia and $E$. coli recovered from a Swedish patient admitted in hospital in New Delhi, India .(Kumarasamy et. Al., 2010).

\section{Resistance to Colistin}

Colistin belongs to the antimicrobial class of polymyxin and acts by binding to the lipid a moiety of the bacterial lipopolysaccharide and subsequently disintegrating the bacteria membrane. Resistance to colistin associated with lipopolysacharide modification leading to a high level of antibiotic non-susceptibility. A detail review of various resistance 
mechanisms to polymyxin has recently been published. (Falagas ME ET. Al. ,2010).

\section{Resistance to Tigecycline}

Tigecycline is first glycylcycline to be approved by the FDA, may lose its activity against MDR strains, particularly when low drug concentration are attained in the serum during treatment. Resistance develops when the MIC of the targeted pathogen exceed the Cmax of the drug, which is almost the rule for all targeted A. baumannii strains. According to molecular studies efflux pumps seem to be the most important mechanism of resistance to tigecycline both in enterobacteriacae and non-fermenting rods. (Keeney D et. Al. ,2007).

\section{Resistance to Fluoroquinolone}

Fluoroquinolone such as ciprofloxacin with bactericidal activity against gram negative pathogens. Fluoroquinolone act by binding to the nuclear enzyme DNA gyrase and topoisomerase IV. In spite of particular targets, resistance mechanisms both chromosomal and plasmidic have been developed. Chromosomal resistance mechanism includes target mutation and augmented expression of efflux pumps. Among plasmid-mediated resistance the most important mechanism are represented by acetylation, efflux pumps and production of fluoroquinilone resistant proteins which protect the target from inhibition. (Drlica K ,2003).

\section{Resistance to Aminoglycosides}

Aminoglycosides such as Gentamycin or Amicacin are compound with bactericidal activity against gram negative pathogens. It inhibits protein synthesis by binding to $16 \mathrm{~S}$ subunit of rRNA and they also disrupt the integrity of the bacterial cell membrane. There are three different mechanisms responsible for resistance to amino glycosides such as over expression of 
efflux pumps target, modification and presence of modifying enzyme. (Dozzo P. ,2008)

\section{Resistance to Phosphonic acid derivatives}

Fosfomycin is a phosphonic acid derivative which inhibits an enzyme involved in the first step of bacteria cellwall synthesis. Resistance to the fosfomycin is rarely reported because of its particular molecular structure and mechanism of action. Therefore fosfomycin is a an important alternative treatment of urinary tract infection including, those cause by MDR pathogens. (Eschenburg S,2005).

\section{COMMON MULTIDRUG RESISTANT URINARY PATHOGENS}

\section{EMBL producing Enterobacteriaceae}

Enterobacteriaceae are the family of gram negative bacteria that includes E. coli, Klebsiella spp., seratia spp., Salmonella spp., among these family B- lactamase production is the most common mechanism of resistance. (Shorr AF ,2009). The ESBL produced by enterobacteraceae have variable resistance to cephalosporins, penicilins, B-lactamase inhibitors and monobactams. ESBL have plasmid encoded genes associate with great ability to spread between bacteria. The ESBL producing phenotypes present in approximately 10-15 \% of enterobacteriaceae. ESBL producing eneterobacteriaceae have traditionally been treated with carbapenems. (Slama ,2008)

\section{Pseudomonas aeruginosa}

Pseudomonas aeruginosa is associated with a variety of infections including urinary tract infection, pneumonia, wound infection, and bacterimia. Pseudomonas has intrinsic antibiotic resistance, has also acquired other mechanism of resistance, including B- lactamase 
carbapenemase, and multidrug efflux pump. Rates of resistance of third generation antibiotics are cephalosporines, cabapenems and quinolones have continued to rise from decade. (Slama ,2008). About $30 \%$ of Pseudomonas aeruginosa exhibited the resistant to cephalosporins, carbapenema, aminoglycosides and fluroquinilones. Now a day the infection with MDR $P$. aeruginosa is treated with colistin.(McGowan JE,2006).

\section{Acinetobacter species}

Acinetobacter baumannii was a MDR nosocomial and community acquired pathogen commonly found in wound infection. The incidence of acinobacter has increase in urinary tract infection, blood stream infections, and surgical infection. Furthermore strains of acinobacter that are resistant to all aminogycosides, cephalosporins, B-latams and fluoroquinilones are increasing in prevalence. (Slama TG ,2008). These MDR strains are problematic to treat and are associated with more frequent relapses. Now a day colistin and tigecycline are used for treatment of MDR Acinetobacter. .(McGowan JE,2006).

\section{APPROCHES TO NEW ANTIBIOTICS}

Antibiotic resistance is a global problem affecting medical and economical condition of the patient. In past decades many kind of resistant strains are discovered such as Methicillin Resistant Staphylococcus aurous (MRSA) (Wagenlehner FME, 2004), Multidrug resistant Pseudomonas aeroginosa (Linuma Y, 2007). Vancomycin resistant Entercocci (VRE) (Gold HS; 2001), Extended spectrum beta-lactamase (ESBL) resistant enterococci. (Bhattacharya S: 2006). Although antibiotic resistance is increases in hospitals and community in both gram positive and gram negative pathogens. To overcome the situation few newly antibacterial agents are approved since 2003. 
Many new antibiotics such as oxazolidinones are being developed for the infection of Gram-positive pathogens. Radezolid and torezolid are currently in phase II of trials and several new members such as delafloxacin, nemonoxacin, zabofloxacin, WCK-771 etc. of this class are under development. (File T et. Al., 2008)

Many other different antibiotics are in different phases of trials such as CEM-101 ，BC-3205,RWJ-416457, AFN-1252, ACHN-490， CB-182804, Friulimicin are in phase-I of trials ; Amadacycline , Delafloxacin, Nemonoxacin, WCK-771,Zabofloxacin,PZ-601,NXL-103,NXL-104= Ceftazidine, CXA-101,Ramoplanin are in phase-II of trials and Oritavancin, Dalbavancin,Iclaprim, Ceftobiprole,Difimicin are in phase-III of trials. (Mariani R. et, al., 2005)

\section{CONCLUSION:}

Antibiotic therapy is a choice of treatment for the bacterial infections , but due to development of multidrug resistant strains the antibiotics do not effective as a treatment to patient. New antibiotic developed which may be the used as a antibiotic therapy in future in resistant cases. The present review is helpful to know the multidrug resistant pathogens are sensitive to particular antibiotics and the proper antibiotic has been administrated to patient for rapid cure of infection.

\section{REFERRENCES :}

Bhattacharya S:2006. ESBL from petridish to patient. Indian J. Medi. Microbol ; 24:20-24)

Brakhage AA, Al-Abdallah Q, Tuncher A, Sprote P. Evolution of B- lactam biosynthesis genes and recruitment of transacting factors . Phytochemistry,2005, 66(11),1200-1210. 
Dozzo P, Moser HE. New aminiglycoside antibiotics.Expert Opin. Ther. Path. ,2008, 20(10), 1321-1341.

Drlica K, Malik M, Fluroquinolones : action and resistance. 2003 , Curr. Top. Med. Chem. 3(3) ,249-282.

Eschenburg S, Priestman M, Schonbrunn E. Evidence that the fosfomycin target Cys 115 in UDP-N-acetylglucosamineenolpyruviltranferase (MurA) is essential for product release. J. bio. Chem. ,2005,280(5) ,3757-3763.

Falagas ME,Rafailidis PI ,Mathaiou DK. Resistance to polymyxin genes : Mechanisms , frequency and treatment option. Drug resist. Update, $2010,13(4-5), 132-138$.

File T, Bagheri F,Bush L. A phase study comparing two doses of radezolid to linezolid in adult with uncomplicated skin and skin structure infections (uSSSI). 48 th Intersci. Conf. on Antimicrob. Agent Chemother,2008; No. L-1515c.

Gold HS. Vancomycin resistant entercocci : mechanism and clinical observations. Clin. Infect. Disease ,2001;;33:210-219)

Hooton TM , Stamm WE. Management of acute uncomplicated urinary tract infection in adults. Med Clin North Am. 1991; 75:339-357.

Infectious Disease Society of America .The 10*12 initiative : Pursuing a global commitment to develop 10 new antibacterial drugs by 2020. Clin. Infect. Dis. $2010,50(8), 1081-1083$

Jacobson SH . Afive year prospective followup of women with non-obstructive pyelonephritic renal scarring. Scand J Urol Nephrl. ,1991;25:51-53. 
Keeney D , Ruzin A,Bradford PA, Ram A. Transcriptional regulator , AcrAB , an RND type efflux pump are associated with decreased susceptibility to tigecycline in enterobactor cloacae. Microb. Drug Resist.,2007, 13(1) , $1-6$.

Kumarasamy KK, Tolemen MA, Walsh TR . Emmergence of new antibiotic resistance mechanism in India, Pakistan and UK : A molecular biological and epidemiological study. Lancet infec. Dis. ,2010, 10(9) ,597-602.

Linuma $Y$. Infection control strategies for antimicrobial resistance, Nippon Rinsho ,2007; 65 : 175-184.

McGowan JE Jr. Resistance in non-fermenting gram negative bacteria : multidrug resistance to the maximum. Am. J. Med. ,2006,11916 Suppl. 1), S29-S36.

Marani R , Granata G,Maffioli S, Serina S, Brunati C, Sosio M,Marazzi A, Vanini A, Patel D, White R. Antibiotics GE23077, novel inhibitors of bacteria RNA polymerase. Part 3 : chemical derivatization. Bioorg.med. chem.. ,2005: $15: 3748-3752$.

Rawat D, Nair D. Extended spectrum B-lactamases in gram-negative bacteria. J Glob.infect Dis. ,2010,2(3),263-274

Slama TG. Gram negative antibiotic resistance: there is a prize to pay. Crit care. $2008: 12$ (Suppl. 4):S4.

Shorr AF. Review of studies of the impact on gram negative bacterial resistance on outcomes in the intensive care unit. Crit. Care Med. ,2009: 37(4), 1463-1469.

Stamm WE ; Norrby SR . Urinary tract infection disease panorama and chalanges. J Infect Des, 2001; 183:S1-4). 
International Journal of Researches in Biosciences, Agriculture \& Technology

September 2013

Issue-1, Volume-1
ISSN No. (Online):

2347-517X

Stuart B. Factors impacting on problem of antibiotic resistance. J Journal of antimicrobial chemotherapy, 2002: 49, 1, 25-30

Wagenlehner FME and KG Naber. New drugs for gram-positive uropathogens. J. Antimicrobial agents; 2004; 24:S39-43.) 